\title{
Microwave Background Constraints on Decaying Defects
}

\author{
Robert H. Brandenberger ${ }^{1,2}$, Brandon Carter ${ }^{3}$ and Anne-Christine Davis ${ }^{4}$ \\ 1 TH Division, CERN, 1211 Geneva 23, Switzerland (address from 9/15/01 - 3/15/02); \\ ${ }^{2}$ Physics Department, Brown University, Providence, R.I. 02912, USA, \\ e-mail: rhb@het.brown.edu; \\ ${ }^{3}$ LUTH, Observatoire de Paris-Meudon, 92 195 Meudon, France, \\ e-mail: carter@obspm.fr; \\ ${ }^{4}$ DAMTP, Center for Mathematical Sciences, University of Cambridge, \\ Wilberforce Road, Cambridge, CB3 OWA, U.K., \\ e-mail: A.C.Davis@damtp.cam.ac.uk.
}

(15 February 2002)

\begin{abstract}
Embedded defects are predicted in a host of particle physics theories, in particular in the standard electroweak theory. They can be stabilized by interactions with the cosmological plasma, but will decay once the plasma falls out of equilibrium, emitting a substantial fraction of non-thermal photons. If the decay happens after a redshift of about $10^{6}$, these photons will give rise to spectral distortions of the Cosmic Microwave Background, which leads to strong constraints on the underlying particle physics theory. Such constraints apply to any model with decaying defects, and in particular to theories predicting decaying vortons, thereby leading to constraints stronger than the dark matter limit.
\end{abstract}

PACS numbers: $98.80 \mathrm{Cq}$

\section{INTRODUCTION}

It has recently been realized [1] that many types of embedded defects can be stable in the cosmological plasma of the early Universe. Particular examples are the Zstring [2] of the standard electroweak theory and the pion strings of the Sigma model description of low energy QCD in the limit of vanishing pion mass [3], which are both stabilized by interactions with the electromagnetic plasma. Since the photons fall out of thermal equilibrium at the time $t_{\mathrm{ls}}$ of last scattering, the stabilization forces will disappear at this time and the embedded defects will decay, emitting a certain fraction of their energy as non-thermal photons.

Cosmological models with non-thermal photon production occurring after a redshift of $10^{6}$ are strongly constrained by the precision measurements 跑 of the blackbody spectrum of the Cosmic Microwave Background (CMB). The fraction of photons produced after the above redshift must be smaller than $7 \times 10^{-5}$, otherwise the Compton y parameter measuring the spectral distortion of the CMB would be larger than the observational limits.

The purpose of this Letter is to study the constraints on theories with embedded defects which result from the FIRAS limits. Our analysis is applicable to any theory with decaying topological defects. As we will see, the strongest constraints result for theories with vortons [5]. The resulting constraints are in many cases stronger than the nucleosynthesis and dark matter constraints on vorton models obtained in previous work [6:7].

The outline of this Letter is as follows. We first review why some embedded defects can be stabilized by the cosmological plasma of the early Universe. We then focus on models with embedded strings, such as the standard electroweak theory, or decaying topological strings and derive the constraints in several circumstances, depending on whether the strings have current and form vortons or not, and depending on whether they decay during the string scaling regime or in the friction-dominated regime. In the final section we discuss our results.

\section{STABILIZATION AND DECAY OF EMBEDDED DEFECTS}

To illustrate the mechanism that renders some embedded defects stable in the early Universe, we shall consider a model [8] in which the order parameter consists of four real scalar fields with a standard symmetry breaking potential symmetric in the four fields. We will assume that two of the fields are electrically charged and the other two neutral, as is the case in the standard electroweak theory and in the Sigma model description of low energy QCD in the limit of vanishing pion mass.

In an electromagnetic plasma, the interactions with the photon bath will lead to an effective potential for the order parameter which breaks the symmetry between the charged and the neutral fields, while preserving the symmetry within the neutral scalar field sector. The potential is lifted more in the direction of the charged scalar fields. Thus, the vacuum manifold becomes $S^{1}$, giving rise to cosmic string solutions of the full field equations which look like the standard $U(1)$ cosmic string configuration of the neutral fields with the charged scalar fields set to zero.

The thermal averaging implicit in the above analysis breaks down after the time of last scattering, and thus 
it is expected that the confining potential will disappear and the embedded strings will decay, emitting a fraction $f$ (which is expected to be smaller but of the order of 1) of its energy as photons. Since the topological defects are out-of-equilibrium objects, the photons produced by their decay will lead to spectral distortions of the thermal CMB 冈

Let us now look at this stabilization mechanism in more detail. The Higgs sector of our model is described by the following Lagrangian

$$
\mathcal{L}=\frac{1}{2}\left(\chi_{\mid \mu}{ }^{*} \chi^{\mid \mu}+\phi_{; \mu}{ }^{*} \phi^{; \mu}\right)-V
$$

with the symmetry breaking potential

$$
V=\frac{\lambda}{4}\left(\chi^{*} \chi+\phi^{*} \phi-\eta^{2}\right)^{2},
$$

and with gauge-covariant derivative

$$
\chi_{\mid \mu}=\chi_{; \mu}+i e A_{\mu} \chi,
$$

where $e$ is the fundamental charge, $A_{\mu}$ is the gauge field of electromagnetism, and a semicolon denotes the spacetime covariant derivative. The field $\phi$ denotes the neutral Higgs doublet, the field $\chi$ the charged Higgs doublet. Using the language of the low-energy Sigma model of QCD we can write

$$
\chi=\pi_{1}+i \pi_{2}, \quad \phi=\pi_{3}+i \pi_{0} .
$$

The finite temperature corrections to the potential of the theory given by (11) were worked out in detail in [8]. Keeping only the contributions to the finite temperature effective potential $V_{\Theta}$ quadratic in the temperature $\Theta$ we obtain

$$
\begin{aligned}
V_{\Theta}-V & =\frac{\Theta^{2}}{2} e^{2}\left(\frac{1}{6} A_{\mu} A^{\mu}+\pi \chi^{*} \chi\right) \\
& +\Theta^{2} \frac{\lambda}{4}\left(\chi^{*} \chi+\phi^{*} \phi-\frac{2}{3} \eta^{2}\right) .
\end{aligned}
$$

The second term in the first line is responsible for the breaking of the degeneracy in the potential between the charged and neutral scalar fields. Equation (5) describes a potential which is lifted by different amounts in the neutral and charged scalar field directions compared to the zero temperature potential. For temperatures below the critical temperature $\Theta_{c}=\sqrt{2} \eta$, the space of lowest energy states forms a manifold $S^{1}$ consisting of configurations with $\chi=0$ and $\phi^{*} \phi=\eta_{\Theta}^{2}$, where $\eta_{\Theta}$ is given by

\footnotetext{
* Note that the non-thermal nature of the decay products of strings has recently been invoked as a possible source of non-thermally distributed cold dark matter 9].
}

$$
\eta_{\Theta}^{2}=\eta^{2}-\frac{\Theta^{2}}{2}
$$

Thus, there is a static string solution consisting of a $U(1)$ cosmic string in the $\phi$ variables with $\chi=0$, the embedded string. As for usual cosmic strings, the mass per unit length $\mathcal{U}_{0}$ of this embedded string is given by

$$
\mathcal{U}_{0}=\alpha \eta^{2},
$$

where $\alpha$ is a numerical factor of order 1 .

The embedded string is stable in the temperature range immediately below the critical temperature for which the curvature of the potential in the $\chi$ direction is positive at $\chi=0$. This is the case for

$$
\sqrt{2}\left(1+\frac{2 \pi e^{2}}{\lambda}\right)^{-1 / 2}<\frac{\Theta}{\eta}<\sqrt{2} .
$$

When the temperature drops below the lower limit given in (8), the string does not disappear, but rather undergoes a core phase transition [10] (see also [11,12]) in which the charged field $\chi$ acquires a non-vanishing value $\chi_{\Theta}$ in the string core which lowers the potential energy density in the core. What results is an asymmetric vortex defect which, as realized in [8], will generically be superconducting, the superconductivity being induced by the phase gradient of $\chi$ in the string core. The value of $\left|\chi_{\Theta}\right|$ is given by

$$
\chi^{*} \chi=\eta^{2}-\frac{\Theta^{2}}{2}\left(1+\frac{2 \pi e^{2}}{\lambda}\right) .
$$

The potential energy density $V_{\Theta}$ in the core of an asymmetric vortex defect is smaller than the corresponding energy density $V_{\Theta, 0}$ of the symmetric embedded defect. Combining the bare potential (2) with the quadratic temperature corrections given by (5) we find the following expression for the finite temperature effective potential $V_{\Theta}$

$$
\begin{aligned}
V_{\Theta} & =e^{2} \frac{\Theta^{2}}{2}\left(\frac{1}{6} A_{\mu} A^{\mu}+\pi \chi^{*} \chi\right) \\
& +\frac{\lambda}{4}\left(\chi^{*} \chi+\phi^{*} \phi-\eta_{\Theta}^{2}\right)^{2}+C_{\Theta},
\end{aligned}
$$

where $C_{\Theta}$ is a constant depending on temperature.

The potential energy density $\Delta V_{\Theta}$ in the core of the asymmetric embedded defect is obtained by evaluating (10) at the center of the core, i.e. for $\phi=0$ and $\chi$ given by (9), and subtracting the constant $C_{\Theta}$. Let us now consider temperatures $\Theta$ much lower than the critical temperature $\Theta_{c}$. In this case, the result is

$$
\Delta V_{\Theta} \simeq \frac{e^{2}}{2} \pi \Theta^{2} \eta^{2}
$$

The radius $r_{\chi}$ of the asymmetric vortex is given by equating the potential energy density and the tension energy within the core radius, with the result 


$$
r_{\chi} \sim \frac{\left(\chi^{*} \chi\right)^{1 / 2}}{\Delta V_{\Theta}^{1 / 2}} \simeq\left(\frac{2}{\pi}\right)^{1 / 2} e^{-1} \Theta^{-1} .
$$

For small gauge coupling constant, the width of the asymmetric vortex thus turns out to be larger than the thermal length, thus justifying our use of thermal averaging to study the local vortex structure. Combining (11) and (12), we see that the energy per unit length $\mathcal{U}$ is of the same order of magnitude as $\mathcal{U}_{0}$, namely

$$
\mathcal{U} \sim \eta^{2} .
$$

This demonstrates that most of the energy loss of embedded defects occurs when they finally decay.

Since the asymmetric vortices are superconducting, string loops will generically form vortons [5]. The strings acquire their current at the time $\Theta_{Q}$ of the core phase transition, i.e. $($ see $(8))$

$$
\Theta_{\mathrm{Q}}=\sqrt{2}\left(1+\frac{2 \pi e^{2}}{\lambda}\right)^{-1 / 2} \eta .
$$

Since this temperature is only slightly lower than the temperature at which the strings initially form, the string network will still be in the friction-dominated phase when the current condensation occurs. This information is crucial (see [6]) for determining the vorton density.

In the following, we will use the knowledge of the energy contained in embedded vortices, in particular those of asymmetric core nature, to determine the cosmological constraints.

\section{CONSTRAINTS}

Cosmic vortices (and other defects) that decay into (in part) photons at some temperature $\Theta_{\mathrm{d}}$ corresponding to a redshift of less than $10^{6}$ will produce spectral distortions of the CMB and will thus be strongly constrained by the COBE/FIRAS data 顿. The constraint on the nonthermal fractional energy density production in photons is

$$
\frac{\delta \rho_{\gamma}}{\rho_{\gamma}} \lesssim 7 \times 10^{-5}
$$

This constraint arises from the limits to the Compton $y$ parameter which measures the spectral distortion. Any defects which decay after a redshift of $10^{6}$ will be subject to these FIRAS constraints. Thus, the following considerations will apply to stabilized embedded defects and to decaying topological defects (such a decay might be induced by a late time phase transition).

The specific bounds on defect models will depend on the type of defect, on the density of defects and on the specifics of the decay. We will focus on cosmic strings, both of topological and of embedded type. First, we consider strings which are in their scaling regime at the time of decay (see e.g. 13] for a review of cosmic string dynamics). This applies, for example, to embedded vortices formed at early times and in which superconductivity is absent or too weak to produce vortons. It also applies to topological, non-superconducting strings formed at early times. If the strings are formed later than some critical time (whose value is derived below), the string network will not yet be scaling and the dynamics will still be friction-dominated. Since the density of strings is higher in this phase relative to a string scaling configuration, the bounds in this case are different. This is the second case we treat. Finally, we analyze the - from the point of view of embedded asymmetric vortices most realistic - case of a gas of vortons produced by the superconducting string loops. Cosmological constraints on models with vortons have previously been studied (see e.g. [6,7] and references therein). The constraints we derive here are tighter (but obviously only apply in the case of decaying vortons such as those of interest in the case of embedded defects).

\section{A. Scaling Cosmic Strings}

First let us examine the case of cosmic strings in the scaling regime. Cosmic strings formed at a temperature $\Theta_{c}$ with a mass per unit length of $\mathcal{U}$, which is approximately $\Theta_{c}^{2}$, will be in the scaling regime after a period of time $t_{\mathrm{x}}$, where [14]

$$
t_{\mathrm{x}} \approx(G \mathcal{U})^{-1} t_{c} \approx\left(\frac{45}{32 \pi^{3}}\right)^{1 / 2}\left(\frac{m_{\mathrm{P}}}{\eta}\right)^{4} m_{\mathrm{P}}^{-1}
$$

where $\mathcal{U} \approx \eta^{2}$. In the scaling regime the density of strings at time $\mathrm{t}$ is

$$
\rho_{\mathrm{s}}=\nu \frac{\eta^{2}}{t^{2}}
$$

where the constant $\nu$ determines the number of long string segments per Hubble volume, and whose value is $\nu \sim 10$ (see 13 and references therein).

Let us first consider strings decaying at the time $t_{\mathrm{ls}}$ of last scattering. From (17) it follows that the density of strings at last scattering is

$$
\rho_{\mathrm{s}}=\nu \frac{32 \pi^{3}}{45} \frac{z_{\mathrm{eq}}}{z_{\mathrm{ls}}} \frac{\eta^{2}}{m_{\mathrm{P}}^{2}} \Theta_{\mathrm{ls}}^{4},
$$

where $z_{\mathrm{eq}}$ is the redshift at the time of equal matter and radiation. If a fraction $f$ of the energy of the strings goes into photons, then the COBE/FIRAS constraint (15) becomes

$$
\frac{f \rho_{\mathrm{s}}}{\rho}=f \nu \frac{32 \pi}{3}\left(\frac{\eta}{m_{\mathrm{P}}}\right)^{2} \frac{z_{\mathrm{eq}}}{z_{\mathrm{ls}}} \lesssim 7 \times 10^{-5} .
$$

This yields a constraint on the scale of symmetry breaking

$$
\frac{\eta}{m_{\mathrm{p}}} \lesssim\left(7 \times 10^{-5}\right)^{1 / 2}\left(\frac{z_{\mathrm{ls}}}{z_{\mathrm{eq}}} \frac{3}{32 \pi}\right)^{1 / 2} f^{-1 / 2} \nu^{-1 / 2} .
$$


For example, assuming that $f$ is of order 1 then (20) results in the constraint

$$
\eta \lesssim \nu^{-1 / 2} 10^{16} \mathrm{GeV} .
$$

Thus, for values of $\nu$ in the range indicated by present cosmic string simulations, the COBE/FIRAS constaint severely constrains decaying cosmic string models with a symmetry breaking scale given by the scale of Grand Unification. Note, however, that all cosmic string models with this scale of symmetry breaking are already constrained 15 17] by the fact that they would produce observable isocurvature fluctuations in the CMB of amplitude larger than what is compatible with recent $\mathrm{CMB}$ anisotropy observations.

Consider now strings decaying at a redshift $z_{\mathrm{d}}$ larger than $z_{\text {eq }}$ (but smaller than $10^{6}$ ). In this case, the COBE/FIRAS constraint yields a result analogous to (20), but without the factor of $\left(z_{\mathrm{ls}} / z_{\mathrm{eq}}\right)^{1 / 2}$ (a factor resulting from the faster redshifting of the radiation energy density after $t_{\text {eq }}$ compared to that of the string energy density). Thus, the bound is weaker by a factor of about 3 than the bound given in (21). Conversely, if the strings decay after $t_{\mathrm{ls}}$, the bound is stronger by a factor of $\left(z_{\mathrm{d}} / z_{\mathrm{ls}}\right)^{1 / 2}$, a fact which again follows immediately from the different rates of redshifting of energy densities.

\section{B. Friction Dominated Strings}

Non-conducting cosmic strings will remain in the friction dominated phase in which they strongly interact with the surrounding plasma until the time $t_{\mathrm{x}}$ given in (16), which corresponds to the Kibble limit temperature given by

$$
\Theta_{\mathrm{x}}=\left(\frac{32 \pi^{3}}{45}\right)^{1 / 4} m_{\mathrm{P}}\left(\frac{\eta}{m_{\mathrm{P}}}\right)^{2}
$$

This friction domination condition will still be satisfied at the time when the strings decay (assumed to be $t_{\mathrm{ls}}$ ) whenever $\Theta_{\mathrm{x}}$ is less than $\Theta_{\mathrm{ls}}$, i.e. less than $10^{-13} \mathrm{GeV}$. This gives

$$
\frac{\eta}{m_{\mathrm{P}}} \lesssim 10^{-16}
$$

i.e. $\eta \lesssim 10^{3} \mathrm{GeV}$. As well as applying to non-conducting strings, Kibble's lower bound (22) is also relevant for strings with currents of the non-electromagnetic chiral type [7], but for electrically conducting strings the epoch of friction domination can be greatly prolonged 18 20, and in consequence the limit (23) will be considerably relaxed.

The network of strings in the friction epoch can be described as a Brownian walk network with scaling length given by [13, 6 ]

$$
L\{\Theta\} \approx m_{\mathrm{P}}^{1 / 2} \frac{\Theta_{c}}{\Theta^{5 / 2}} .
$$

The corresponding energy density in the string network is

$$
\rho_{s}\{\Theta\} \sim \nu \frac{\mathcal{U}}{L\{\Theta\}^{2}} \sim \nu m_{\mathrm{P}}^{-1} \Theta^{5} .
$$

Note that the scale of symmetry breaking $\eta$ has cancelled out. This is due to the fact that as $\eta$ increases, the mass per unit length of each string increases, but the string density decreases because we are considering later times in the friction epoch. From the $\Theta^{5}$ scaling of $\rho_{s}$ it follows immediately that strings in the friction epoch are not constrained by the COBE/FIRAS data, unless they lead to vortons.

Even if they contained no currents, strings formed at the electroweak limit would not be excluded by the limit (23). However, electroweak strings will have quark and lepton zero modes in their core [21] and are thus currentcarrying. Current-carrying strings give rise to stationary loops, or vortons, and will therefore be subject to the constraints discussed in the next section.

\section{Vortons}

If any cosmic strings, either embedded strings or topologically stable strings, are current-carrying, then string loops would form a stationary equilibrium state or vorton [5]. For topological strings, the vortons could be very stable, particularly in the chiral case [7]. In earlier work [6] we have considered constraints on particle physics theories yielding vortons from nucleosynthesis and dark matter considerations. If the vortons survive at least a few minutes, then a constraint can be derived by demanding that the Universe remain radiation dominated at the time of nucleoysynthesis. If the vortons are absolutely stable or decay with a lifetime greater than the age of the Universe, an additional and stronger constraint follows by requiring that the vorton density does not overclose the Universe.

The main point of the present work is to consider the additional constraint arising from the limit (15) provided by the COBE/FIRAS data 佂 in cases for which vortons decay between a redshift of $10^{b}$ and today, thus giving rise to decay products that would produce observable distortions of the black body spectrum.

If the string becomes current-carrying at a time $\Theta_{Q}$ that is in the friction phase of the string dynamics, then the vorton density at temperature $\Theta$ is given by [6]

$$
\rho_{\mathrm{v}}=\tilde{f}\left(\frac{\Theta_{\mathrm{Q}}}{m_{\mathrm{P}}}\right)^{5 / 4}\left(\frac{\Theta_{\mathrm{Q}}}{\Theta_{c}}\right)^{3 / 2} \Theta_{\mathrm{Q}} \Theta^{3}
$$

where $\tilde{f}$ is a constant of the (rough) order of 1 . This result holds both in the radiation and matter dominated phases.

As before, we denote the temperature at which the vortons decay by $\Theta_{\mathrm{d}}$. If the vortons emit a fraction $f$ 
of their energy as photons, then the fractional photon energy density input from vorton decay is

$$
\frac{\Delta \rho_{\gamma}\left\{\Theta_{\mathrm{d}}\right\}}{\rho_{\gamma}\left\{\Theta_{\mathrm{d}}\right\}}=\kappa\left(\frac{\Theta_{\mathrm{Q}}}{m_{\mathrm{P}}}\right)^{5 / 4}\left(\frac{\Theta_{\mathrm{Q}}}{\Theta_{c}}\right)^{3 / 2} \frac{\Theta_{\mathrm{Q}}}{\Theta_{\mathrm{d}}},
$$

in which the constant $\kappa$ is given by

$$
\kappa=\frac{30}{\pi^{2} g_{*}\left\{\Theta_{\mathrm{d}}\right\}} f \tilde{f}
$$

where, $g_{*}$ denotes the number of spin degrees of freedom in the radiation bath. The COBE/FIRAS constraint (15) thus becomes

$$
\kappa\left(\frac{\Theta_{\mathrm{Q}}}{m_{\mathrm{P}}}\right)^{5 / 4}\left(\frac{\Theta_{\mathrm{Q}}}{\Theta_{c}}\right)^{3 / 2} \frac{\Theta_{\mathrm{Q}}}{\Theta_{\mathrm{d}}}<7 \times 10^{-5} .
$$

As a first example, assume that $\Theta_{\mathrm{Q}}=\Theta_{c}$ (which, as shown in (14), gives the right order of magnitude for embedded strings), and that the decay occurs at the time of last scattering (as again is reasonable for the embedded strings which are stabilized by electromagntic plasma effects), i.e. $\Theta_{\mathrm{d}}=\Theta_{\mathrm{ls}}$. In this case, using the estimate $\kappa \sim 1$, the general constraint (29) leads to

$$
\Theta_{c} \lesssim 10^{5} \mathrm{GeV} .
$$

If we relax the assumption that $\Theta_{\mathrm{Q}}=\Theta_{c}$ and instead parametrize the current condensation time by

$$
\frac{\Theta_{Q}}{\Theta_{c}}=10^{\alpha},
$$

where $\alpha$ is some (negative) number, the constraint 29) becomes

$$
\kappa\left(\frac{\Theta_{c}}{m_{\mathrm{P}}}\right)^{5 / 4} \frac{\Theta_{c}}{\Theta_{d}}<7 \times 10^{-5} 10^{-(15 / 4) \alpha},
$$

which corresponds to a severe relaxation of the constraint on $\Theta_{c}$.

\section{DISCUSSION}

We have considered cosmological constraints on models with decaying topological defects which result from demanding that the photons produced in the decay do not lead to spectral distortions of the CMB in excess of the observational limits from the COBE/FIRAS experiment. The strongest limits arise for theories leading to decaying vortons. Any theory giving rise to vortons resulting from a string forming phase transition above $10^{5} \mathrm{GeV}$, and which decayed from a redshift of $10^{6}$ to today, would produce a too large spectral distortion as measured by the Compton $y$ parameter, and thus be ruled out. The above constraint applies both to vortons resulting from topological strings or those resulting from embedded strings which have become stabilised by plasma processes. These constraints are in fact stronger than constraints on vorton models requiring compatibility with nucleosynthesis and with the dark matter abundance limits $[6]$.

Since many types of embedded strings are stabilized by interactions with the electromagnetic plasma, undergo core phase transitions and become superconducting, thus yielding vortons [8] which decay at the time of last scattering, our constraints are very important for theories with embedded defects.

Our analysis also gives rise to constraints on theories with non-superconducting topological cosmic strings with GUT scale symmetry breaking scale, provided they decay in the relevant redshift interval.

\section{Acknowledgements}

Two of us (ACD and RB) wish to thank W. Unruh and A. Zhitnitsky for hospitality at U.B.C., Vancouver, where this work was initiated. This work was supported in part by the ESF COSLAB programme and by a Royal Society-CNRS exchange grant (BC,ACD), by PPARC (ACD), by the US Department of Energy under Contract DE-FG0291ER40688, Task A (RB), and by an Accord between CNRS and Brown University (BC,RB). We are grateful to Herb Fried for securing this Accord.

[1] M. Nagasawa and R. H. Brandenberger, Phys. Lett. B 467, 205 (1999) arXiv:hep-ph/9904261.

[2] T. Vachaspati and A. Achucarro, Phys. Rev. D 44, 3067 (1991).

[3] X. Zhang, T. Huang and R. H. Brandenberger, Phys. Rev. D 58, 027702 (1998) arXiv:hep-ph/9711452.

[4] D. Fixsen et al., Astrophys. J. 473, 576 (1996) arXiv:astro-ph/9605054.

[5] R. L. Davis and E. P. Shellard, Nucl. Phys. B 323, 209 (1989).

[6] R. H. Brandenberger, B. Carter, A. C. Davis and M. Trodden, Phys. Rev. D 54, 6059 (1996) arXiv:hep$\mathrm{ph} / 9605382$.

[7] B. Carter and A. C. Davis, Phys. Rev. D 61, 123501 (2000) arXiv:hep-ph/9910560

[8] B. Carter, R. H. Brandenberger and A. C. Davis, "Thermal stabilisation of superconducting sigma strings and their drum vortons," arXiv:hep-ph/0201155.

[9] R. Jeannerot, X. Zhang and R. H. Brandenberger, J.H.E.P. 9912, 003 (1999) arXiv:hep-ph/9901357.

[10] M. Axenides and L. Perivolaropoulos, Phys. Rev. D 56, 1973 (1997) arXiv:hep-ph/9702221.

[11] M. Axenides, L. Perivolaropoulos and M. Trodden, Phys. Rev. D 58, 083505 (1998) arXiv:hep-ph/9801232.

[12] M. Axenides, L. Perivolaropoulos and T. N. Tomaras, Phys. Rev. D 58, 103512 (1998) arXiv:hep-ph/9803355.

[13] A. Vilenkin and E.P.S. Shellard, Cosmic strings and other topological defects (Cambridge Univ. Press, Cambridge, 1994). 
[14] T. W. Kibble, Acta Phys. Polon. B 13, 723 (1982).

[15] A. Albrecht, "Defect models of cosmic structure in light of the new CMB data," in XXXVth Rencontres de Moriond: Energy Densities in the Universe (2000) arXiv:astro-ph/0009129.

[16] R. Durrer, M. Kunz and A. Melchiorri, "Cosmic structure formation with topological defects," arXiv:astro$\mathrm{ph} / 0110348$.

[17] J. Magueijo and R. H. Brandenberger, "Cosmic defects and cosmology," arXiv:astro-ph/0002030.

[18] K. Dimopoulos and A. C. Davis, Phys. Rev. D 57, 692701 (1998) arXiv:hep-ph/9705302

[19] K. Dimopoulos and A. C. Davis, Phys. Lett. B 446, 236246 (1999) arXiv:hep-ph/9901250

[20] B. Carter, R. H. Brandenberger, A. C. Davis and G. Sigl, JHEP 0011, 019 (2000) arXiv:hep-ph/0009278.

[21] A. C. Davis and W. B. Perkins, Phys. Lett. B 390, 107 (1997) arXiv:hep-ph/9610292. 